\title{
ESTUDO DA RELAÇÃO ENTRE LINEAMENTOS ESTRUTURAIS E A EXPLORAÇÃO DE ÁGUA SUBTERRÂNEA POR MEIO DA ANÁLISE DE FAVORABILIDADE
}

\section{STUDY OF RELATION OF STRUCTURAL LINEAMENTS AND GROUNDWATER EXPLORATION THROUGH FAVORABILITY ANALYSIS}

\author{
Alexandre Campane Vidal ${ }^{1}$, Sidnei Pires Rostirolla ${ }^{2}$, \\ Chang Hung Kiang ${ }^{3}$ e Maria Luiza Martini ${ }^{2}$
}

\begin{abstract}
Recebido em: 23/09/2006; aceito em: 16/12/2006
RESUMO A Análise de Favorabilidade foi aplicada como um método para avaliar a correlação entre as feições estruturais e o comportamento de fluxo do aqǘf́rero. A análise foi realizada em área aflorante do Grupo de Tubarão, entre as cidades de Campinas e Sorocaba (Estado de São Paulo). Essa área foi escolhida devido à sua complexidade geológica e à baixa previsão do comportamento de fluxo antes da perfuração. Para se aplicar este método, foram consideradas como produtivas as regiões com valores de elevada capacidade específica. Ao todo foram obtidas seis variáveis por meio da análise de imagens de satélite Landsat 7-ETM, Modelos Digitais do Terreno (SRTM), e da utilização de mapas geológicos da área. Os resultados demonstram que a região entre as cidades de Elias Fausto e Monte Mor apresenta a melhor correlação com o lineamento estrutural, o que demonstra ser este um fator importante para o alto potencial exploratório do aquífero nesta área.
\end{abstract}

Palavras-chave: análise de favorabilidade, hidrogeologia, Grupo Tubarão.

\begin{abstract}
Favorability analysis has been applied as a method to evaluate the correlation between the structural lineament and flow behavior in aquifers. The analysis was carried out in the outcropping areas of Tubarão Group, between the cities of Campinas and Sorocaba (São Paulo State, Brazil). The study area was chosen because of its geological complexity and the consequent lower predictability of well performance before drilling. To apply this method, we considered as "mineralized" areas those regions with highest specific capacity values, taking also into account six types of variables interpreted from radar images Landsat 7-ETM, Shuttle Radar Topography Mission (SRTM) and structural geological maps. The results reveal that the region encompassing between the cities of Elias Fausto and Monte Mor presents the best correlation with structural lineation which can be a factor in the high potential for groundwater exploration in this area.
\end{abstract}

Keywords: favorability analysis, hydrogeology, Tubarão Group.

\section{INTRODUÇÃO}

A definição dos principais fatores responsáveis pela melhor vazão nos poços, bem como a delimitação de áreas com mesmas características de fluxo do aqüífero, é importante para o gerenciamento dos recursos hídricos. Esse conhecimento se torna fundamental em áreas pouco exploradas e com baixa densidade de informação.

A melhor condição do aqüífero está vinculada a variáveis relacionadas principalmente aos fatores geológicos, tais como permeabilidade e porosidade da rocha, presença de falhas e fraturas, conectividade dos corpos arenosos, além de outros fatores. O número de variáveis aumenta quando as informações aplicadas para avaliar as condições de fluxo são oriundas de informações de poços.
Devido ao grande número de variáveis envolvidas na caracterização de aqüíferos, tornase necessária a utilização de métodos estatísticos que possibilitem avaliar o peso de cada variável para a melhor previsão de seu comportamento.

Várias áreas da exploração mineral aplicam métodos estatísticos que tem por objetivo a delimitação da ocorrência mineral, com base na associação de algumas variáveis. Em muitos casos, a estatística bayesiana é utilizada por meio da análise de favorabilidade (OTT et al. 2006, ROSTIROLLA et al. 2003, HARBAUGH et al. 1995).

A análise de favorabilidade é uma metodologia estatística que tem sido utilizada para indicar a existência de depósitos minerais, embasada na ponderação e associação espacial de dados geológicos e geofísicos. O propósito desta

\footnotetext{
${ }^{1}$ UNICAMP/IG/Departamento de Geologia e Recursos Naturais (vidal@ige.unicamp.br)

${ }^{2}$ UFPR/ Departamento de Geologia (rostirolla@ufpr.br),(maria@ufpr.br)

${ }^{3}$ UNESP/IGCE/Departamento de Geologia Aplicada (chang@rc.unesp.br)
} 
avaliação é discriminar as regiões com melhores probabilidades para descobertas, possibilitando priorizar sub-áreas para trabalhos de prospecção (ROSTIROLLA 1996).

Com base na distribuição espacial e na coexistência entre variáveis e depósitos, o sistema de avaliação proposto torna possível quantificar a associação de cada variável mapeada com o processo mineralizador $\mathrm{e}$, conseqüentemente, o grau indicativo da existência de um bem mineral.

O método estatístico bayesiano consiste em determinar a probabilidade de ocorrer um dado evento, mediante um condicionante, como por exemplo a probabilidade de ocorrer um depósito a partir da existência de alguns atributos mapeados.

$\mathrm{Na}$ exploração de água subterrânea, a estatística bayesiana é muitas vezes utilizada para análise de incertezas de parâmetros hidrológicos de pequena escala, visando à calibração de modelos de simulação de fluxo (FEYEN et al. 2002, 2003).

A análise de favorabilidade foi aplicada por Vidal et al. (2006), para avaliar o potencial de exploração da água subterrânea para a região de exposição do Subgrupo Itararé no Estado de São Paulo, entre os municípios de Campinas e Sorocaba. Nesse estudo, as variáveis utilizadas foram espessura de arenitos, salinidade da água subterrânea, cota altimétrica do terreno e mapa de lineamentos principais. $\mathrm{O}$ estudo realizado apontou a variável "lineamentos principais" como de menor peso em relação às outras variáveis utilizadas.

No entanto, toda análise estrutural da região de estudo é prejudicada pela falta de afloramentos e ausência de informações de subsuperfície. Além disso, no trabalho de Vidal et al. (2006), foram utilizadas informações de imagens de satélite Shuttle Radar Topography Mission (SRTM) apenas para a definição de lineamentos principais e, em muitos casos, essa fonte de dados possibilita diferentes produtos que podem auxiliar na análise estrutural.

O objetivo deste trabalho é aplicar a análise de favorabilidade a uma base de dados estruturais obtidos de imagens de satélite Landsat 7-ETM, Modelos Digitais do Terreno (SRTM), para avaliar sua relação com as áreas que apresentam as melhores características de fluxo. Isso contradiz os modelos conceituais e a prática de exploração na região, tendo em vista o controle exercido pelas estruturas tectônicas na dinâmica do aquífero. Em virtude da aparente dicotomia entre os pesos calculados para os lineamentos e a constatação qualitativa de que os mesmos consistem em variáveis diagnósticas importantes para identificar áreas com boas condições de fluxo do aqüífero. Por essa razão optou-se por proceder a uma análise de imagens mais detalhada.

Como área de estudo, foi definida a região aflorante do Sistema Aqüífero Tubarão, na porção central do Estado São Paulo, a mesma área referente ao trabalho de Vidal et al. (2006), que é caracterizada por baixa condutividade hidráulica e fraco potencial produtivo (DIOGO et al. 1981, DAEE, 1981a, b).

\section{ÁREA DE ESTUDO}

A área de estudo perfaz cerca de $4.350 \mathrm{~km}^{2}$ da região aflorante do Grupo Tubarão no Estado de São Paulo. A região, situada entre os paralelos $22^{\circ} 45^{\prime}$ e $23^{\circ} 20^{\prime}$ de latitude sul, é limitada a leste pelas rochas do embasamento cristalino, a oeste pelos sedimentos do Grupo Passa Dois (Figura 1).

O Grupo Tubarão é formado por rochas sedimentares neopaleozóicas da Bacia do Paraná. No Estado de São Paulo é formado pelo Subgrupo Itararé, constituído por rochas depositadas em condições glaciais, e pela Formação Tatuí, sobreposta, constituída por rochas de origem pós-glacial.

Os sedimentos do Subgrupo Itararé estão presentes na quase totalidade da área de pesquisa, sendo que a Formação Tatuí ocorre em estreita faixa a oeste.

Considerando dados de toda a Bacia do Paraná, França; Potter (1989) adotam a terminologia litoestratigráfica de Supergrupo Tubarão, dividido em Grupo Itararé e Grupo Guatá. No Estado de São Paulo, a unidade estratigráfica Itararé permanece indivisa, recebendo a denominação de Subgrupo, pela maioria dos autores.

Os problemas encontrados para a subdivisão do Subgrupo Itararé devem-se à falta de continuidade lateral dos corpos rochosos e de camadas-guia nesta unidade. Esta situação está condicionada, em parte, à variabilidade dos ambientes de sedimentação deste conjunto de rochas (FRANÇA; POTTER 1989, CAETANOCHANG 1984, FÚLFARO et al. 1984).

Segundo Stevaux et al. (1987), o comportamento errático dos sedimentos arenosos, a distribuição irregular dos poços e o pequeno número de informações em relação à elevada heterogeneidade, são as principais causas para a alta incerteza na prospecção de água subterrânea na região. Além disso, os depósitos do Grupo Tubarão são cortados, em diversos pontos, por intrusões de diabásio que localmente, ou mesmo regionalmente, interrompem a continuidade da 
rocha reservatório.

Com base na distribuição de arenitos, o Subgrupo Itararé no Estado de São Paulo pode ser dividido em três unidades: inferior e superior, mais arenosas e com espessura aproximada de 200m cada uma; e unidade intermediária, onde predominam sedimentos finos, com espessura de 200 a 500 m (DAEE-UNESP 1979).

A porção inferior, predominantemente arenosa, ocorre em estreita faixa junto ao embasamento da bacia, intercalando arenitos conglomeráticos e ritmitos. A unidade intermediária, de maior extensão, ocorre no centro da área aflorante e apresenta diamictitos de matriz lamítica, arenitos finos e pelitos. A unidade superior apresenta maior ocorrência junto à borda oeste, constituída principalmente por arenitos grossos e diamictitos de matriz arenosa.

A geometria dos aqüíferos é influenciada, em escala regional, pela disposição das unidades arenosas presentes no Subgrupo Itararé. Segundo a classificação de Diogo et al. (1981), as unidades superior e inferior apresentam as melhores condições de exploração.

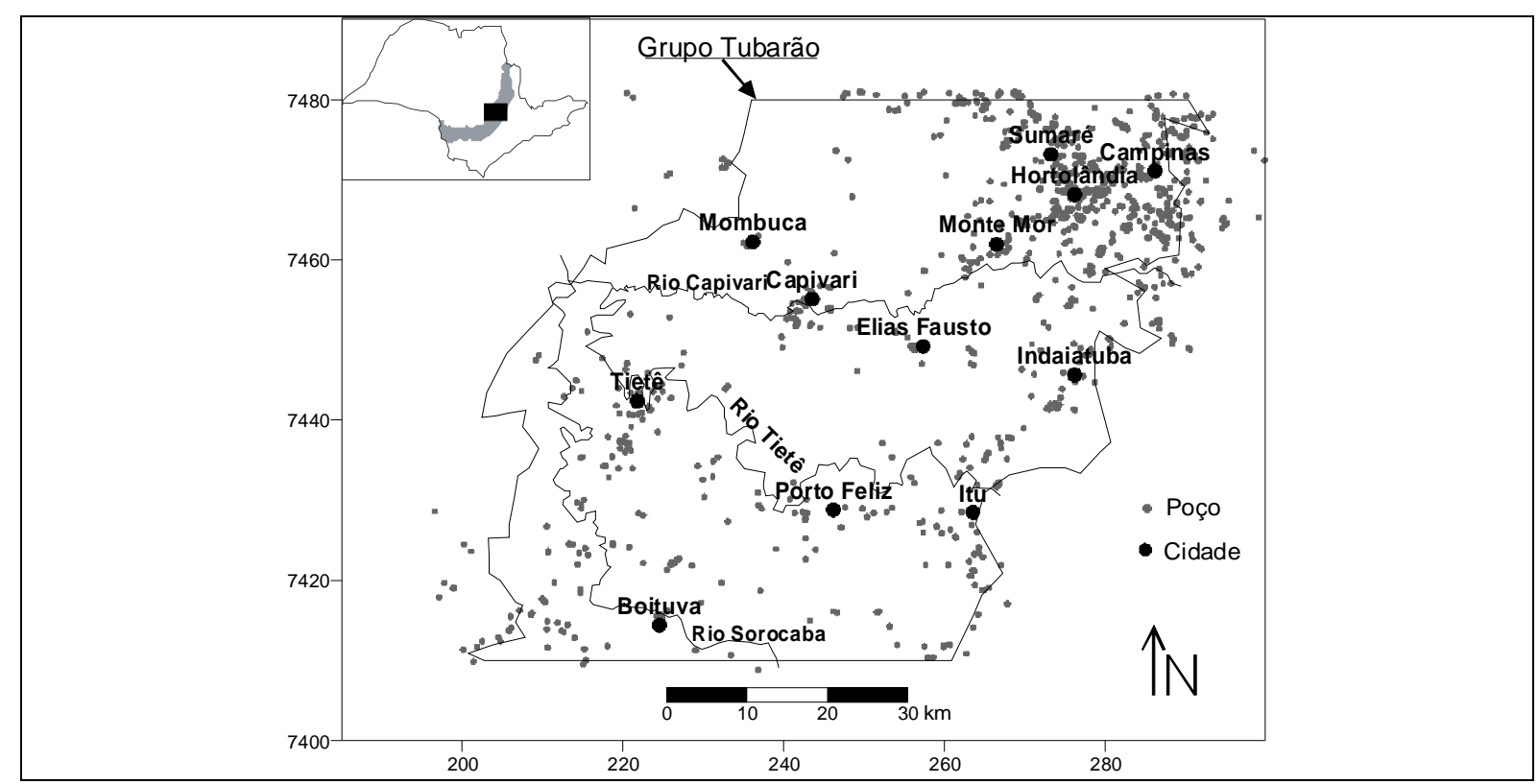

Figura 1- Mapa de localização, com indicações dos 978 poços utilizados neste estudo. No alto, à esquerda, está indicada a área de ocorrência do Grupo Tubarão no Estado de São Paulo e o limite da área de estudo (em preto)

Figure 1-Location map with indications of wells used in this study. In the top, to the left, is indicated the occurrence area of the Group Tubarão in São Paulo State and the limit of the study area (in black)

Steavux et al. (1987) analisaram o Subgrupo Itararé na bacia do Rio Capivari e sugeriram um modelo para prospecção de água subterrânea, determinando três tipos de aqüíferos: (1) arenitos conglomeráticos e arenitos grossos de leques aluviais, com vazões entre 1 e $5 \mathrm{~m}^{3} / \mathrm{h}$; (2) sedimentos arenosos de frente deltaica, com vazões médias de $35 \mathrm{~m}^{3} / \mathrm{h}$; (3) sedimentos arenosos de geometria tabular e de grande extensão, com vazões que chegam até $100 \mathrm{~m}^{3} / \mathrm{h}$.

Estudos detalhados das condições de fluxo do aquífero foram realizados por vários autores (YOSHINAGA-PEREIRA 1996, IG/SMA 1995, LOPES 1994, IG/SMA 1993). Estes trabalhos apontam a grande variabilidade da capacidade específica, com valores médios que variam de 0,09 a $1,32 \mathrm{~m}^{3} / \mathrm{h} / \mathrm{m}$, e ressaltam a influência dos corpos de diabásio sobre o comportamento do fluxo dos aqüíferos. Além disso, YoshinagaPereira (1996) destaca que a maior produtividade dos poços está relacionada às movimentações neotectônicas, evidenciada pelos maiores valores de capacidade específica nos poços situados nos lineamentos de drenagem.

\section{MÉTODOS}

$\mathrm{Na}$ análise de favorabilidade os atributos georeferenciados são discretizados em células e correlacionados espacialmente. A combinação das informações de diversos níveis é utilizada para gerar uma saída de dados que permite calcular a probabilidade de determinada ocorrência de uma acumulação. Descrição detalhada da análise de Favorabilidade está presente nos trabalhos de Rostirolla (1996) e Bonham-Carter (1994).

A etapa inicial da avaliação corresponde à montagem do modelo de ocorrência, quando são discriminadas as evidências favoráveis à concentração (critérios diagnósticos). A segunda 
etapa consiste na geração de um modelo de probabilidade, estruturado segundo o modo de ocorrência. Assim, é possível predizer a favorabilidade de um depósito ou de uma acumulação, de tamanho e volume adequados, ocorrer em determinada área-alvo.

Seguindo as premissas presentes em Rostirolla et al. (1997), são consideradas "necessárias" aquelas variáveis que sempre existem nos depósitos conhecidos, enquanto as variáveis "suficientes" podem ou não existir, mas sua ocorrência representa um forte indício de mineralização. A condição de suficiência de uma variável $E$ é satisfeita quando a probabilidade de existência do depósito (hipótese $H$ ) é maximizada com a presença da variável $(\mathrm{P}(H \mid E)=$ máximo $)$. A condição de necessidade da variável é satisfeita quando a probabilidade de não-existência do depósito é maximizada com a ausência da variável $(\mathrm{P}(\bar{H} \mid \bar{E})=$ máximo).

Nos mapas de variáveis exploratórias, os pontos conhecidos (amostras da população estudada) são tratados como pixels e estimados espacialmente. A probabilidade condicional é obtida pelo número de pixels da variável inserido na área mineralizada, em relação ao número total de pixels existente da variável.

Segundo o modelo adotado por BonhamCarter (1994), em todas células avaliadas, a chance posterior $C h\left(H \mid E_{j}^{k}\right)$ de ser encontrado um depósito (ou hipótese $H$ ) é dada pelo somatório da chance prévia $\mathrm{Ch}(H)$, obtida da razão entre o número de pixels da área mineralizada pelo número de pixels da área de estudo, com os ponderadores $w_{j}^{k}$ referentes às evidências $E_{j}^{k}$ :

$$
\ln C h\left(H \mid E_{j}^{k}\right)=w_{j}^{k}+\ln C h(H)
$$

Onde o índice superior $k$ refere-se à presença (valor positivo) ou ausência (valor negativo) da variável, e os ponderadores $w_{j}^{k}$ são razões de probabilidade (likelihood ratios). As razões de probabilidade são calculadas do seguinte modo para cada variável $E_{j}^{k}$ :

$$
w_{j}^{k}=\ln \frac{P\left(E_{j}^{k} \mid H\right)}{P\left(E_{j}^{k} \mid \bar{H}\right)}=\ln \frac{\frac{H \cap E_{j}^{k}}{H}}{\frac{\bar{H} \cap E_{j}^{k}}{\bar{H}}}
$$

Todos os níveis georreferenciados adicionais devem ser computados como mapas binários e, para $E_{j}(j=1,2,3, \ldots \mathrm{n})$ mapas, o somatório é representado pela fórmula:

$$
C h\left(H \mid E_{1}^{k} \cap E_{2}^{k} \cap \ldots \cap E_{n}^{k}\right)=\exp \left\{\sum_{j=1}^{n} w_{j}^{k}+\ln C h(H)\right\}
$$

Baseado nos resultados, a probabilidade posterior (ou favorabilidade para cada célula avaliada) pode ser obtida a partir da chance posterior (equação 3).

Para aplicação da análise de favorabilidade na exploração de água subterrânea, foram adotadas como condições de sucesso as áreas de maior produtividade nos poços, verificadas a partir dos valores de capacidade específica (obtidas quando a vazão é dividida pela diferença entre o nível dinâmico e o nível estático). Esta medida corresponde à quantidade de água retirada do poço por unidade de tempo e de rebaixamento.

A variável capacidade específica é influenciada pelas características físicas do reservatório, traduzida essencialmente pela transmissividade do aqüífero. Neste trabalho, a incerteza associada à variável capacidade específica não será avaliada.

Para a confecção dos mapas necessários à análise de favorabilidade, foi utilizada a base de dados presente no trabalho de Vidal (2002) e informações adicionais adquiridas em empresas de perfuração de poços. Os poços da base de dados apresentam distribuição irregular, com maior concentração na região nordeste e próximo aos principais municípios da área de estudo (Figura 1). Esta base conta com a catalogação de 978 poços com dados de vazão, nível estático e nível dinâmico.

A etapa inicial para o desenvolvimento da análise de favorabilidade foi a padronização dos mapas em malha 2D, com $220 \times 220$ blocos e dimensões de cada bloco de $450 \times 350 \mathrm{~m}$. A malha adotada foi determinada com essas dimensões para definir, com exatidão, as feições obtidas da interpretação das imagens, pois as variáveis digitalizadas possuem comportamento discreto, representadas na forma de pontos.

Com relação à caracterização estrutural, poucos são os trabalhos específicos sobre esse assunto na região de estudo e, muitas vezes, estes focam áreas restritas. Importante estudo foi realizado pelo DAEE-UNESP (1979) na área aflorante do Subgrupo Itararé no Estado de São Paulo, definindo zonas de falhas em toda área de estudo e, por isso, as informações referentes a esse mapa foram incorporadas como uma variável na análise de favorabilidade.

As outras variáveis foram definidas por meio da análise de imagens de satélite Landsat 7-ETM, Modelos Digitais do Terreno (SRTM). A superposição de eventos pode ser identificada pela análise dos produtos de sensores remotos, onde são observados comportamentos diferenciados nos conjuntos litológicos e 
concentração de elementos estruturais em direções específicas. A análise morfoestrutural iniciou-se com o traçado de lineamentos em imagens SRTM em escalas 1:750.000 e 1:300.000, posteriormente refinadas com a interpretação de imagens Landsat 7 em escalas 1:300.000 e 1:100.000, resultando em feições estruturais representativas de zonas de falhas regionais.

Com a base de dados obtida, foram utilizados diferentes métodos para interpretação das imagens. Os lineamentos estruturais foram traçados sobre feições retilíneas, sendo classificados em três classes: Lineamentos Principais (LP), que apresentam feições claras e expressivas; Lineamento Principal Aparente (LPA), para os casos em que a continuidade é menos evidente; Lineamento Secundário (LS), quando não há rigorosa constância na orientação e continuidade.

Os alinhamentos não foram associados a fatores geológicos, sendo delimitados neste caso por feições de relevo e classificados como principal (AP) e secundário (AS), conforme a expressividade das feições identificadas.

Importante processamento é referente à transferência das informações interpretadas das imagens de satélite para a malha definida para os mapas. $\mathrm{O}$ processo de digitalização das estruturas é realizado ao inserir, em uma base de dados, uma série de pontos georeferenciados da estrutura identificada. A principal limitação desse método é a diferente densidade de pontos na digitalização dos mapas, pois pode ocorrer que entre dois pontos de uma estrutura linear existam células da malha não incorporadas na base de dados. Para solucionar esse problema, foram utilizadas funções específicas contidas em softwares de digitalização de imagens, que visam adensar o número de pontos nas linhas traçadas.

Os mapas utilizados como variáveis nesse trabalho foram: Lineamentos Principais (LP); Lineamentos Principais Aparentes (LPA); Lineamentos Secundários (LS); Alinhamento Principal (AP); Alinhamento Secundário (AS); Distribuição de Falhas, produto do mapeamento DAEE-UNESP (DU). Os mapas das variáveis são apresentados na figura 2 .

As estruturas identificadas foram representadas como linhas e, em muitos casos, representam zonas de falhas que possuem um raio de influência e, portanto, não podem ser representadas por uma linha.

Por isso, para avaliar o raio de influência das feições interpretadas foi desenvolvido um algoritmo para gerar novos pontos a partir das informações originais. A geração dos pontos ocorre em todas as direções, conforme o espaçamento definido. Para os maiores espaçamentos são incorporados os dados gerados nos menores espaçamentos

O comprimento do espaçamento é de $200 \mathrm{~m}$ a cada passo do programa, até atingir a distância máxima de $1.400 \mathrm{~m}$ do traçado original da linha. Assim, entre a linha original e o maior espaçamento há o incremento dos pontos definidos nos espaçamentos anteriores, o que permite elevada densidade de dados e certificação de que todas as células da malha presente no intervalo do espaçamento foram identificadas. Como exemplo, estão representados os mapas de Lineamento Principal Aparente (LPA) para diferentes espaçamentos (Figura 3). 

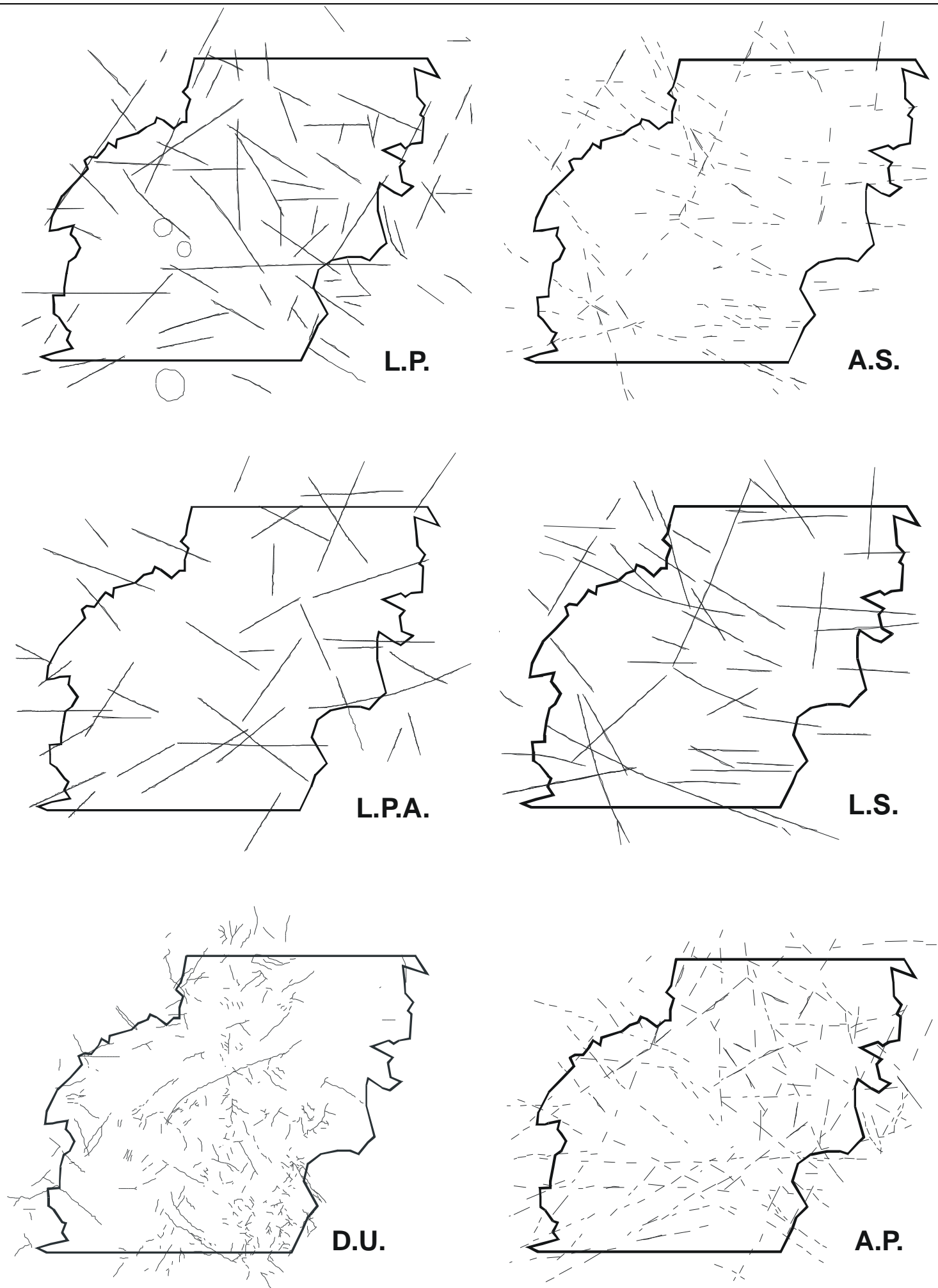

Figura 2- Mapas das variáveis utilizadas na análise de favorabilidade. LP (Lineamentos Principais), AS (Alinhamento Secundário), LPA (Lineamento Principal Aparente), LS (Lineamentos Secundários), DU (distribuição das falhas realizadas no mapeamento DAEE-UNESP 1979), AP (Alinhamento Principal)

Figure 2- Variables maps used in favorability analysis. LP (Principal Lineament), AS (Secondary Alignment), LPA (Lineament Principal Apparent ), LS (Lineament Secondary), DU (faults distribution accomplished in the mapping DAEEUNESP 1979), AP (Main Alignment) 


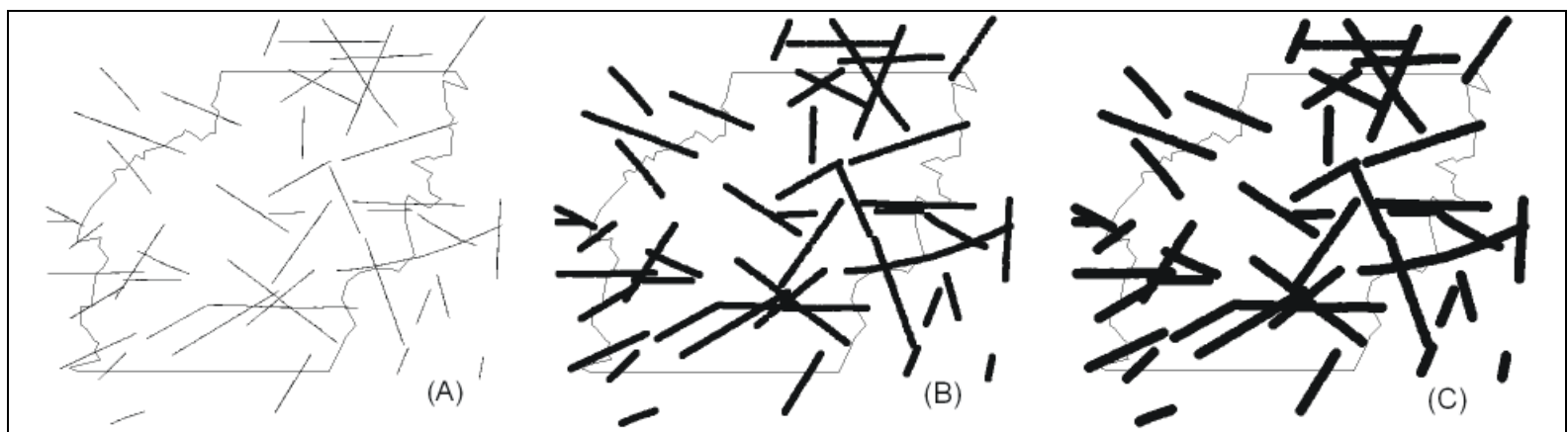

Figura 3- Resultado do programa de aumento do espaçamento para a variável LPA, (A) dados originais, (B) espaçamento de 600 e (C) espaçamento de $1.400 \mathrm{~m}$. A maior espessura da linha é reflexo do agrupamento de alta densidade de pontos

Figure 3- Results of computer routine of increasing of the distance to the variable LPA, (A) original data, $(B)$ spacing of 600 and $(C)$ spacing of $1400 \mathrm{~m}$. The bigger thickness of line is reflex of the grouping of high density of points

\section{RESULTADOS E DISCUSSÕES}

Diferente dos depósitos mineralizados, a exploração de água subterrânea não apresenta um valor de teor de corte (cut off) para um poço se tornar economicamente viável, pois os poços são construídos para diferentes finalidades. Por exemplo, uma vazão de $10 \mathrm{~m}^{3} / \mathrm{h}$ pode ser inviável para o abastecimento público de uma cidade, mas útil para uma pequena propriedade agrícola.

Como não há limites estabelecidos para a definição do teor de corte, estes valores foram determinados pela escolha de medidas estatísticas de localização da distribuição de cada variável (por exemplo, mediana, quartis).

Com base nos dados disponíveis, a variável capacidade específica apresenta distribuição lognormal, com média de $0,37 \mathrm{~m}^{3} / \mathrm{h} / \mathrm{m}$, mediana de $0,12 \mathrm{~m}^{3} / \mathrm{h} / \mathrm{m}$ e variância de 1,06 . Para a adoção das áreas definidas como produtivas, foi estabelecido valor de corte de $0,29 \mathrm{~m}^{3} / \mathrm{h} / \mathrm{m}$, que representa o terceiro quartil da distribuição dos dados de capacidade específica, ou seja, os $25 \%$ maiores valores. Estes valores são também superiores aos valores médios encontrados em outros trabalhos (YOSHINAGA-PEREIRA 1996; IG/SMA 1993, 1995; LOPES 1994).

Para a confecção do mapa foi adotado o método geoestatístico da krigagem como algoritmo interpolador. Para tanto efetuou-se, preliminarmente, a análise variográfica que indicou como mais apropriado o variograma omnidirecional. Este resultado é ocasionado pela distribuição irregular dos dados, com concentração de poços próxima à região do município de Campinas. O modelo de variograma utilizado foi do tipo esférico, com alcance de $8 \mathrm{~km}$ e patamar de 0,9 . A krigagem utilizada foi a ordinária e o mapa final da área, com capacidade específica superior a $0,29 \mathrm{~m}^{3} / \mathrm{h} / \mathrm{m}$, está representado na figura 4.

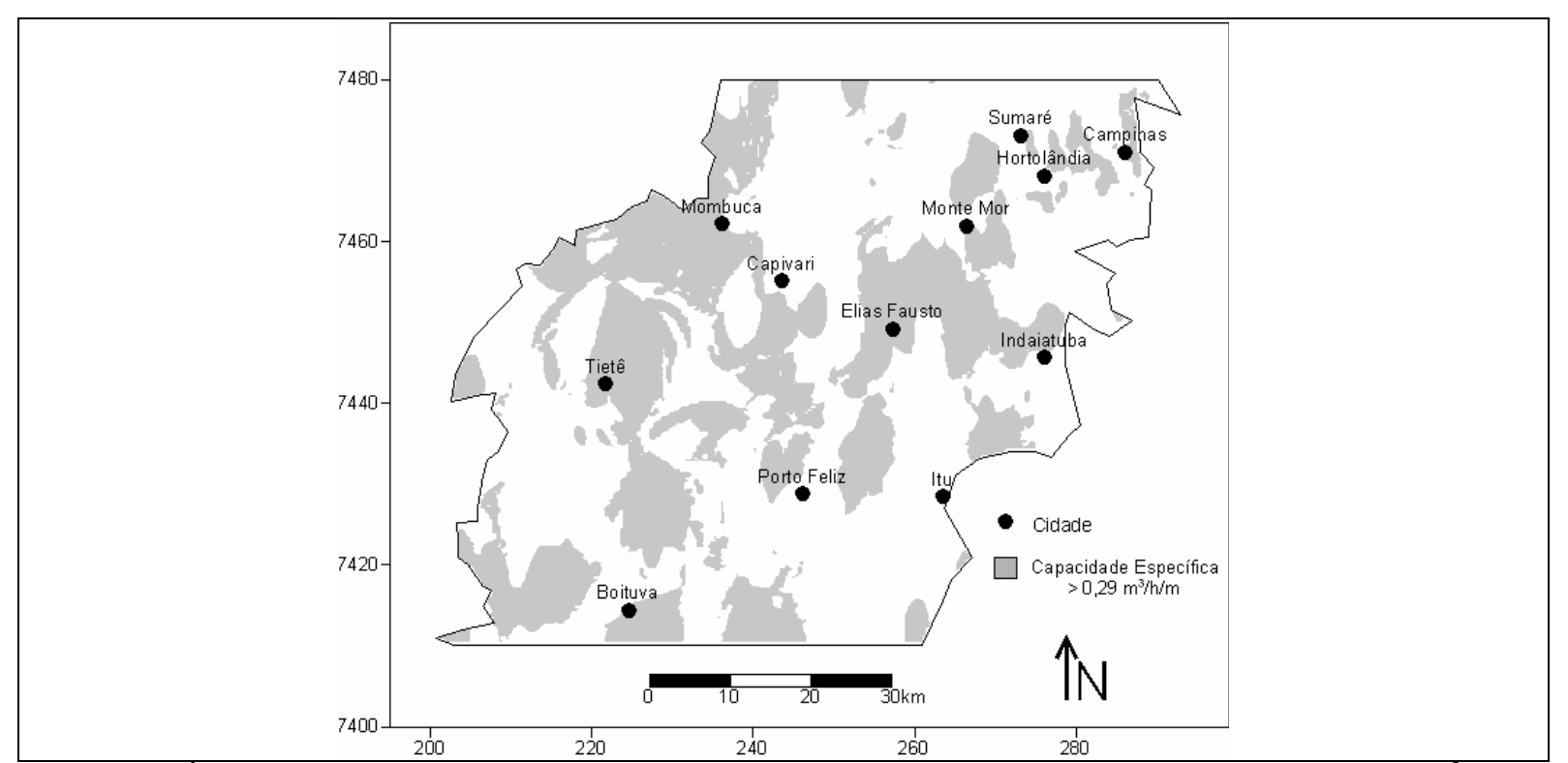

Figura 4- Áreas em coloração cinza representam valores de capacidade específica superiores a $0,29 \mathrm{~m} / \mathrm{h} / \mathrm{m}$, sendo consideradas produtivas pela análise de favorabilidade

Figure 4- Areas in gray color represent values of specific capacity higher than $0,29 \mathrm{~m} / \mathrm{h} / \mathrm{m}$, which are considered productive in the favorability analysis 
No mapa, as áreas produtivas estão distribuídas por toda área de estudo, com destaque para a porção entre Mombuca-Tietê e entre Elias Fausto-Indaiatuba-Monte Mor. Por outro lado, as porções sudeste (município de Itu), norte e sudoeste, podem ser caracterizadas pelos baixos valores de capacidade específica.

Pode ser inferido um eixo com altos valores de capacidade específica, de direção E-W, presente na porção central da área de estudo e atravessando os municípios de Indaiatuba, Elias Fausto, sul de Capivari e entre Mombuca e Tietê.

As variáveis utilizadas na análise referem-se às interpretações realizadas por meio de imagens de satélite e no mapa geológico da área (DAEEUNESP 1979). Conforme apresentado na figura 2, nesses mapas é possível observar diferenças na densidade de informações, claramente observadas pelo número de traços definidos, bem como pelas diferenças nas orientações das estruturas traçadas.

$\mathrm{Na}$ área de pesquisa, a variável LP apresenta as principais estruturas com direção NW-SE na porção central, direção também representada nos mapas de LS e AS.

Outra feição importante pode ser observada nos lineamentos e alinhamentos de direção E-W, presentes na porção sul da área, e que se destacam nos mapas de LS, AP e LPA. Destacase, no extremo nordeste, a baixa frequiência de estruturas significativas em todos os mapas analisados.
No entanto, interpretações mais detalhadas da distribuição das feições identificadas são prejudicadas pelas diferentes configurações entre os mapas. O problema se torna mais significativo quando se busca avaliar quantitativamente qual dos mapas apresenta maior correlação com o mapa de capacidade específica.

\section{Mapa de Favorabilidade}

Por serem variáveis discretas, a análise se inicia com a atribuição do valor 1 na intersecção das áreas produtivas com as feições definidas nos mapas e valor zero quando não há intersecção.

Para o cálculo dos pesos $\left(\mathrm{w}^{+}\right.$e $\left.\mathrm{w}^{-}\right)$das variáveis, foi utilizada a equação 2 através do algoritmo proposto por Bonham-Carter (1994). Estes pesos são os atributos mais importantes na análise de favorabilidade: quanto maior a diferença entre $\mathrm{w}^{+} \mathrm{e} \mathrm{w}^{-}$ou o contraste "C", maior a correlação da variável com a área de maior capacidade específica.

Os valores de contraste para os dados originais estão presentes na tabela 1 . O melhor valor de contraste é obtido pela variável LP, com valor de 0,107 . Os valores de contraste para os mapas de LP e AP são positivos, mas inferiores aos de LP; a variável LS apresenta valor de contraste próximo a zero. Variáveis como DU e AS apresentam valores negativos, indicando não correlação com as áreas de maiores vazões nos poços.

Tabela 1- Pesos definidos e valor de contraste para os dados originais das variáveis utilizadas Table 1-Weights and contrast values defined for the original data of the used variables in this study

\begin{tabular}{l|c|c|c|c|c|c} 
& LP & LPA & AP & LS & DU & AS \\
\hline W+ & 0,0667 & 0,0365 & 0,0490 & 0,0015 & $-0,1135$ & $-0,0284$ \\
\hline W- & $-0,0401$ & $-0,0214$ & $-0,0291$ & $-0,0008$ & 0,0601 & 0,0159 \\
\hline Contraste & 0,1068 & 0,0579 & 0,0781 & 0,0023 & $-0,1736$ & $-0,0442$ \\
\hline
\end{tabular}

Ao comparar o mapa de capacidade específica com o mapa de LP, é possível observar que a variável LP apresenta lineamentos na região central com direção NW-SE e ausência de informações nas porções norte e sudoeste, o que corresponde a uma boa correlação com as áreas de alto valor de capacidade específica.

O contrário pode ser observado para o mapa DU, que apresenta falhas posicionadas na porção central, mas com direção N-S, ausência de estruturas nas bordas leste e sudoeste da área e, por isso, apresenta um baixo valor de contraste.

Os pesos foram também calculados para todos os raios de influência definidos para as variáveis (Figura 5). Os resultados obtidos apontam um aumento dos valores de contraste com o espaçamento para a maioria das variáveis, embora com elevada variabilidade. 


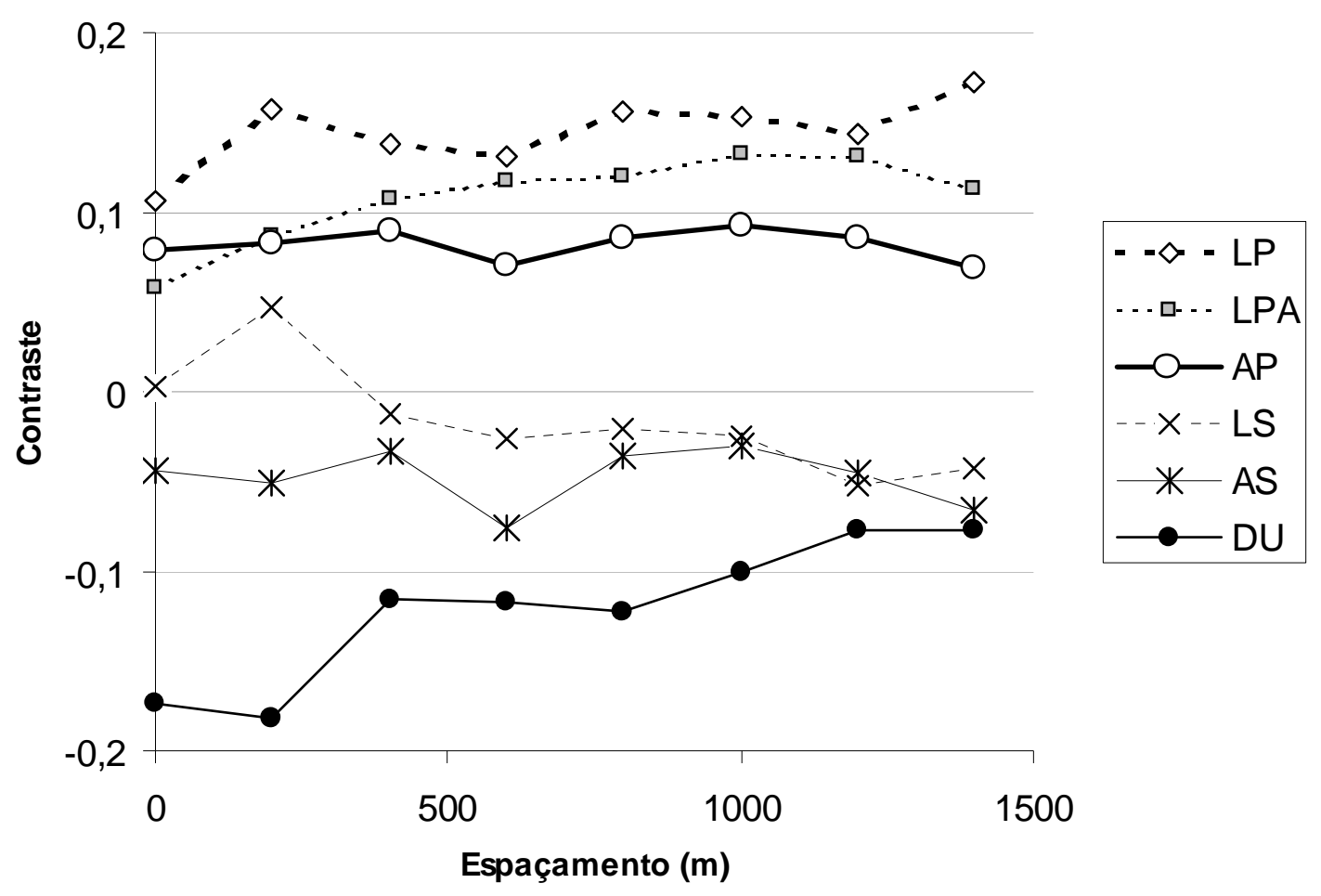

Figura 5- Valores de contraste em relação aos espaçamentos utilizados para avaliar o raio de influência das estruturas identificadas

Figure 5-Contrast values correlated to the distances utilized to evaluate the influence radius of the identified structures

Porém, para todas as variáveis, os valores de contraste foram inferiores a 0,5 , indicando baixa correlação com as áreas produtivas. Isto comprova que nenhuma das variáveis pode ser considerada isoladamente como o principal fator para os altos valores de capacidade especifica na área.

A variável AS apresentou valores de contraste negativos para todos os espaçamentos e não-correlação com o aumento do raio de influência. Também para a variável DU ocorrem valores negativos, mas com o aumento do espaçamento há aumento do contraste.

A variável LS apresenta o maior valor de contraste para o espaçamento de $200 \mathrm{~m}$ e para os maiores espaçamentos a correlação é negativa. A variável AP apresenta todos valores de contraste positivos, porém com baixos valores e pouca variação com o aumento do raio de influência.

Dentre todas, as variáveis LPA e LP apresentaram os melhores resultados, com os máximos valores de contraste acima de 0,1 e com correlação positiva com o aumento do raio de influência. A variável LP apresenta máximo de 0,157 para o raio de pesquisa de $200 \mathrm{~m}$ e a LPA máximo valor de contraste de 0,132 em espaçamento de $1.000 \mathrm{~m}$.
O máximo valor de contraste para um espaçamento específico pode ser considerado um indicador para o raio de influência de uma zona de falha, definida na delimitação dos lineamentos.

$\mathrm{Na}$ confecção do mapa de favorabilidade, foi considerado o raio de influência para cada variável. A determinação de qual espaçamento utilizar foi definido pelo maior valor de contraste crescente a partir dos dados originais. Isso evita a adoção de valores de contraste em grande espaçamento, onde ocorre a descaracterização da distribuição dos pontos em relação às estruturas originais.

Para a confecção do mapa de favorabilidade, foram utilizadas as variáveis com valores de contraste positivos, sendo descartadas as variáveis DU e AS. Assim, o mapa foi elaborado com as seguintes variáveis e espaçamento: LP200 m; LPA-1000 m; AP-400 m; LS-200 m.

A probabilidade posterior foi calculada por meio da soma dos pesos das variáveis para cada célula, mais a probabilidade a priori (Equação 3). Os resultados obtidos de cada célula foram plotados em mapa, que apresentam as áreas mais favoráveis à exploração de água subterrânea correspondente aos maiores valores de probabilidade posterior (Figura 6). 


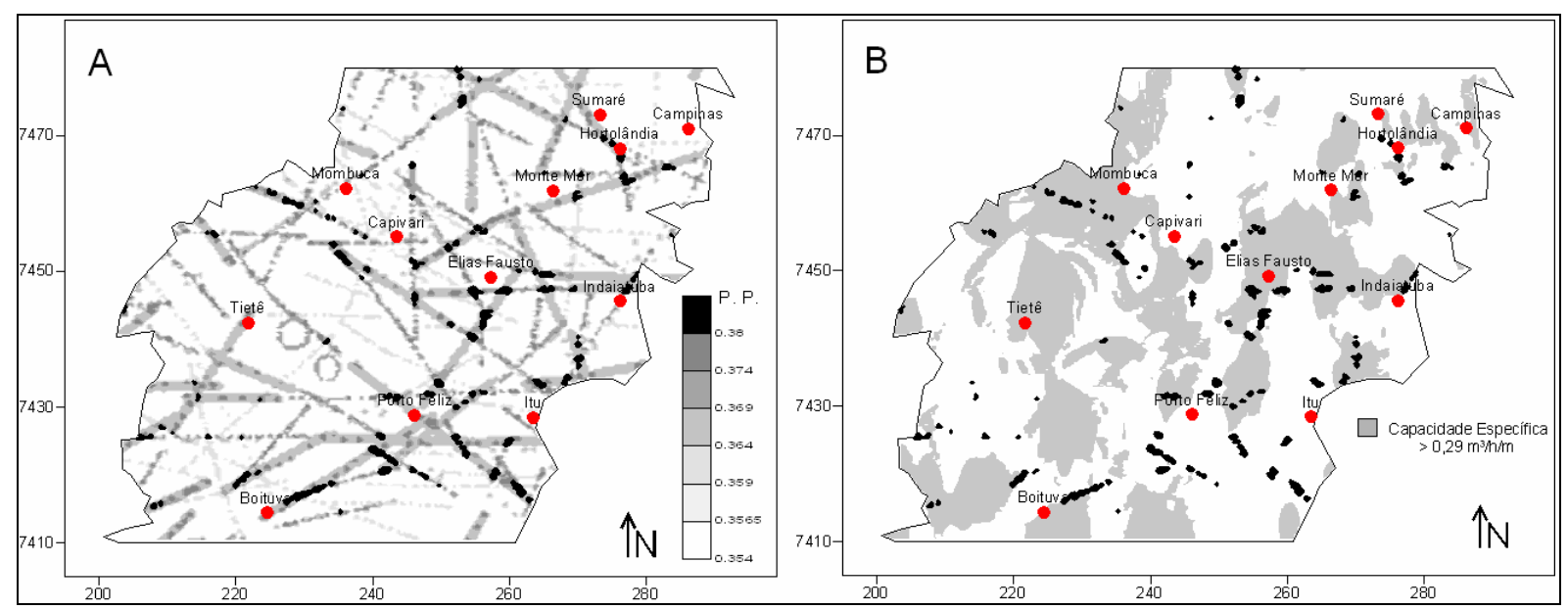

Figura 6- Mapa de favorabilidade para a área de estudo. (A) valores de Probabilidade Posterior (P.P.). (B) Mapa de capacidade específica associada aos maiores valores de Probabilidade Posterior

Figure 6- Favorability map for the study area. (A) map of Posterior Probability values (P.P.). (B) Specific capacity map associated to the higher values of Posterior Probability

De acordo com as variáveis utilizadas, o mapa de favorabilidade indica a maior probabilidade de sucesso relacionada à região de Elias Fausto, Porto Feliz, à região a sul de Capivari e Mombuca, como também à região de Hortolândia e à região entre Boituva e Porto Feliz.

Na região central, claramente se observam as áreas mais favoráveis posicionadas no eixo de direção NW-SE, que incorpora os municípios de Mombuca, Capivari, Elias Fausto e a região entre os municípios de Indaituba e Itu. Este eixo, na região central, foi definido nas quatro variáveis utilizadas na confecção do mapa.

Por outro lado, condições menos favoráveis se encontram nas porções leste, nordeste e ao redor do município de Tietê.

Ao se comparar o mapa de favorabilidade com a capacidade específica, observa-se que as regiões de Elias Fausto, a sul de Capivari e de Mombuca apresentam boa correlação entre os mapas. A análise realizada identifica, de forma parcial, a região de Monte Mor, Sumaré e Hortolândia, como também a região sul entre os municípios de Boituva e Porto Feliz (Figura 6B).

$A$ adoção do valor de raio de influência para a variável LPA, de $1.000 \mathrm{~m}$, pode ser questionada pelo elevado distanciamento. Ao observar a curva de LPA, nota-se discreta inflexão em $600 \mathrm{~m}$, no entanto, não o suficiente para provocar a redução do valor de contraste (Figura 5).

Entretanto, a região de Tietê, com capacidade específica elevada, não foi identificada por este método, indicando a baixa correlação com as estruturas identificadas. Isto está de acordo com os resultados obtidos por Vidal et al. (2006), que indicaram que as melhores condições de fluxo da região de Tietê são reflexo da cota altimétrica baixa e da alta porcentagem de arenitos.

Porém, não é possível a comparação entre os valores obtidos do trabalho de Vidal et al. (2006), pois o mapa de capacidade específica apresenta outra configuração e a malha para os mapas possui diferentes dimensões.

\section{CONCLUSÕES}

O estudo baseado na interpretação de imagens apontou, segundo os critérios definidos, o mapa de Lineamentos Principais (LP) como o que apresentou as melhores correlações com os altos valores de capacidade específica. Por isso, este mapa é o mais recomendado para o uso na definição de áreas com melhores condições aqüíferas.

A análise de favorabilidade aplicada para o aqüífero Tubarão na região aflorante do médio Tietê, indicou boa correlação das feições estruturais com os altos valores de capacidade específica para a região de Elias Fausto. Entretanto, outras áreas com elevada vazão não foram identificadas, como é caso da região de Tietê. As áreas de baixo valor de capacidade específica nas porções nordeste e sudoeste foram confirmadas como de baixo potencial. 


\section{REFERÊNCIAS}

BONHAM-CARTER, G.F. Geographic Information Systems for Geoscientists - Modelling with GIS. Computer Methods in the Geosciences, v.13. $1^{\circ}$ edition, Canada, Pegamon Press, 398p. 1994.

CAETANO-CHANG, M.R., Análise Ambiental e estratigráfica do Subgrupo Itararé (PC) no sudoeste do Estado de São Paulo. 1984. Tese (Doutorado). IG/USP. São Paulo. 1984.

DAEE-UNESP, Mapa Geológico: divisão faciológica do Super-Grupo Tubarão, escala 1:50.000. 1979.

DAEE - Departamento de Águas e Energia Elétrica Estudo de Águas Subterrâneas, Região Administrativa 5 (Campinas), SP. São Paulo, SP. DAEE, 2v. 1981a.

DAEE - Departamento de Águas e Energia Elétrica Estudo de Águas Subterrâneas, Região Administrativa 4 (Sorocaba), SP. São Paulo, SP. DAEE, 2v. 1981b.

DIOGO, A.; BERTACHINI, A.C.; CAMPOS, H.C.N.S.; ROSA, R.B.G.S. Estudo preliminar das características hidráulicas e hidroquímicas do Grupo Tubarão no Estado de São Paulo. In: SIMPÓSIO REGIONAL DE GEOLOGIA, 3; 1981. Curitiba. Atas... Curitiba: SBG, 1981., v.1, p.359-368.

FEYEN, L.; RIBEIRO Jr, P.J.; De SMEDT, F.; DIGGLE, P.J. Bayesian methodology to stochastic capture zone determination: conditioning on transmissivity measurements. Water Resources Research v38 n9 3-12p. 2002.

FEYEN, L.; GOMEZ-HERNANDEZ, P.J.; RIBEIRO Jr, P.J.; BEVEN, K.J.; De SMEDT, F. A bayesian approach to stochastic capture zonedelineation incorporating tracer arrival times, conductivity measurements and hydraulic head observations. Water Resources Research v39 n5 SBH 10. 2003.

FRANÇA, A.B.; POTTER, P.B. Estratigrafia e ambiente deposicional do Grupo Itararé (Permocarbonífero), Bacia do Paraná (Parte 2). Boletim Geociências Petrobrás, v.3 (1/2), p.17-28. 1989.

FÚlFARO, V.J.; STEVAUX, J.C.; SOUZA FILHO, E.E.; BARCELLOS, J.H. A Formação Tatuí (P) no Estado de São Paulo. In: CONGRESSO BRASILEIO DE GEOLOGIA, 22.,1984, Rio de Janeiro. Anais..., Rio de Janeiro: SBG, 1984. v.2, p.711-724.

HARBAUGH, J.W.; DAVIS, J.C.; WENDEBOURG Computing risk for oil prospects: Principles and programs. Computer Methods in the Geosciences, v.14, USA, Pergamon Press, 452p. 1995.

IG/SMA - INSTITUTO GEOLÓGICO, Subsídios do meio físico-geológico ao planejamento do município de Campinas (SP). Relatório Técnico IG/SMA, 3v. 1993.
IG/SMA - INSTITUTO GEOLÓGICO, Subsídios do meio físico-geológico ao planejamento da Porção Média da Bacia do Rio Piracicaba (SP). São Paulo. Relatório Técnico IG/SMA, 4v. 1995.

LOPES, M.F.C. Condições de ocorrência de água subterrânea nas bacias dos rios Piracicaba e Capivari 1994. 83p. Dissertação (Mestrado), UNICAMP. Campinas, 1994.

OTT, N.; KOLLERSBERGER, T., TASSARA, A. GIS analyses and favorability mapping of optimized satellite data in northern Chile to improve exploration for copper mineral deposits. Geosphere v.2(4) p.236252. 2006.

ROSTIROLLA, S.P. Avaliação de favorabilidade em exploração: um enfoque metodológico 1996. 164p. Tese (Doutorado) IGCE/UNESP. Rio Claro. 1996.

ROSTIROLLA, S.P. Alguns aspectos da avaliação de favorabilidade em geologia exploratória. Revista Brasileira de Geociências, v.27(4) p.327-338. 1997.

ROSTIROLLA, S.P.; MATTANA, A.C.; BARTOSZECK, M.K. Bayesian assessment of favorability for oil and gas prospects over the Recôncavo basin, Brazil. AAPG Bulletin v.87, n4 p647-666. 2003.

STEVAUX, J.C.; SOUZA E.E., F' ${ }^{\circ}$ TEIXEIRA, J.A.; LANDIM, P.M.B. Sistemas deposicionais do Subgrupo Itararé (P-C) na bacia hidrográfica do baixo Rio Capivari (SP): um modelo para prospecção de água subterrânea. In: SIMPÓSIO REGIONAL DE GEOLOGIA, 6., 1987, Rio Claro. Atas... Rio Claro: SBG/NSP. 1987, v.1, p. 355-374.

VIDAL, A.C. Estudo hidrogeológico do Aqüífero Tubarão na área de afloramento da porção central do Estado de São Paulo. 2002. 109p. Tese (Doutorado) - IGCE/UNESP, Rio Claro, 2002.

VIDAL, A.C. ; ROSTIROLLA, S.P. ; CHANG, C.H. Análise de Favorabilidade para a exploração de água subterrânea. Revista Brasileira de Geociências, v.35, n.4, p.475-481, 2005.

YOSHINAGA-PEREIRA, S. Proposta de representação cartográfica na avaliação hidrogeológica para o estudo de planejamento e meio ambiente, exemplo da região metropolitana de Campinas-SP. 1996. Tese (Doutorado) - IG/USP, São Paulo 1996.

\section{Agradecimentos}

Os autores expressam agradecimentos aos revisores da Revista Brasileira de Geociências pelas críticas e sugestões ao manuscrito. 
Estudo da relação entre lineamentos estruturais e a exploração de água subterrânea por meio da Análise de Favorabilidade 